\title{
LINHA DE PRODUTO DE SOFTWARE PARA SISTEMAS DE INFORMAÇÃO EM SAÚDE
}

\author{
Software Product Line for Health Information Systems
}

\begin{abstract}
Artur Ziviani'; Antônio Tadeu Azevedo Gomes²; Débora Christina Muchaluat Saade³
\end{abstract}
Resumo Objetivos: Neste documento, apresentamos de forma recaptulativa as atividades de P\&D dos autores na área de sistemas de informação em saúde (SISs) no contexto de sua atuação no Instituto Nacional de Ciência e Tecnologia em Medicina Assistida por Computação Científica (INCT-MACC). Materiais e métodos: Com base na primeira experiência do grupo de desenvolvimento ad hoc de um SIS para suporte remoto ao atendimento médico emergencial de vítimas de infarto agudo do miocárdio (IAM), descrevemos a concepção de uma nova linha de pesquisa que encontra-se atualmente em andamento sobre uma linha de produto de software para SIS. Resultados: Apresentamos detalhadamente a linha de produto de software para aplicações em saúde, chamada SPLiCE (Software Product Line in healthCarE), incluindo os módulos de transversalização de modelos, modelagem de dados clínicos, modelagem arquitetural e geração de código. Conclusão: Dado que são promissoras as perspectivas para o desenvolvimento dirigido por modelos (DDM) para SISs, estamos atualmente elencando as similaridades e diferenças entre SIS de duas famílias principais: emergência pré-hospitalar e vigilância epidemiológica; sobre as quais pretendemos validar inicialmente o SPLiCE como trabalho futuro.

Palavras-chave: Sistemas de informação em saúde, Desenvolvimento dirigido por modelos, Linha de produto de software

Abstract Aims: In this document, we present in a recapitulative way the R\&D activities of the authors in the area of health information systems (HISes) in the context of their participation in the National Institute of Science and Technology in Medicine Assisted by Scientific Computing (INCT-MACC). Materials and methods: Based on the first experience of the group in the ad hoc development of a HIS for the remote support to the emergency healthcare of victims with acute myocardial infarction (AMI), we describe the conception of a new on-going research direction concerning a software product line for HIS. Results: We present in detail the software product line for healthcare (SPLiCE), including its modules for the model transversalization, modeling of clinical data, architectural modeling, and code generation. Conclusion: Given the promising perspectives of model-driven engineering (MDE) for HISes, we are currently eliciting the main commonalities and differences among HISes of two key families: prehospital emergency and epidemiological surveillance, upon which we intend to firstly validate SPLiCE in our future work.

Keywords: Health information systems, Model-driven engineering, Software product line

1. Tecnologista Pleno do Laboratório Nacional de Computação Científica (LNCC/MCTI) - Doutorado em Ciência da Computação, Université Paris 6 - Sorbonne Universités, 2003; 2. Tecnologista Pleno do Laboratório Nacional de Computação Científica (LNCC/MCTI) - Doutorado em Ciência da Computação, PUC-Rio, 2005; 3. Professora Associada da Universidade Federal Fluminense (UFF) - Doutorado em Ciência da Computação, PUC-Rio, 2003. Endereço Eletrônico: zivianni@lncc.br (Artur Zivianni). 


\section{Introdução e Objetivos}

A aplicação de tecnologias de informação e comunicação (TICS) na área de saúde tem sido vista como uma iniciativa promissora por alguns anos e vem progressivamente ganhando espaço recentemente ${ }^{1,2}$. Neste contexto, Cantrill $^{3}$ reflete a respeito dessa iniciativa promissora e sobre os desafios relacionados para alcançá-la, destacando que as suas vantagens potenciais incluem: (i) comunicação aprimorada entre os múltiplos provedores de serviços de saúde de um paciente individual; (ii) eliminação de testes médicos desnecessários; (iii) redução em erros médicos, qualidade aprimorada de atendimento e segurança aprimorada do paciente; e (iv) redução na burocracia e legibilidade aprimorada.

Os últimos anos, entretanto, têm testemunhado um crescimento significativo no ceticismo sobre a eficácia de sistemas de informação em saúde (SISs) no suporte de serviços de saúde mais eficientes ${ }^{4,5}$. Há um entendimento geral que os fatores inibidores para médicos e outros profissionais de saúde, clínicas e hospitais adotarem SISs se concentram em três grupos principais: organizacionais, ambientais e tecnológicos ${ }^{4}$. Em nosso trabalho, focamos nos fatores inibidores tecnológicos, que podem ser resumidos em (de forma alinhada com Raghupathi e Umar ${ }^{5}$ ): (i) custo elevado de desenvolvimento; (ii) falta de padronização aberta; (iii) orientação tecnológica; e (iv) manutenibilidade complexa.

Assim, neste documento, apresentamos de forma recapitulativa as atividades de P\&D dos autores na área de SISs no contexto de sua atuação no Instituto Nacional de Ciência e Tecnologia em Medicina Assistida por Computação Científica (INCT-MACC) ${ }^{6}$. O primeiro projeto do grupo foi o desenvolvimento de forma ad hoc de um sistema de apoio remoto à decisão para casos de infarto agudo do miocárdio $(I A M)^{7}$. Com base na experiência adquirida nesse primeiro projeto, recentemente foi iniciada uma nova linha de trabalho mais abrangente com a proposta de um novo método dirigido por modelos para $\mathrm{SIS}^{8,9}$. Com isso, este documento descreve a experiência anterior de sistema de apoio remoto para atendimento médico emergencial, as lições aprendidas e a nova linha de trabalho que encontra-se atualmente em andamento sobre uma linha de produto de software para SIS.

\section{Materiais e Métodos}

\section{Experiência em Desenvolvimento Ad Hoc de SIS}

A primeira experiência do grupo foi no desenvolvimento ad hoc de SIS e teve a seguinte motivação para área específica. $\mathrm{O}$ infarto agudo do miocárdio (IAM) encontra-se entre as principais causas de óbito e incapacidade física mundialmente ${ }^{10}$. Também é conhecido que a morbidade e a mortalidade de pacientes que sofreram IAM pode ser diminuída pela redução no intervalo de tempo entre a percepção dos primeiros sintomas e o tratamento ${ }^{11}$. Entretanto, em diversos países os chamados programas de Infarto do Miocárdio com Supra de ST (em inglês, ST - Segment Elevation Myocardial Infarction - STEMI) - que dependem de atendimento médico de emergência antecipado (tipicamente, pré-hospitalar) - ainda estão sendo estudados e revistos de maneira a serem implementados eficientemente $^{12}$. Como resultado, pacientes de IAM continuam a experimentar intervalos de tempo mais prolongados até receberem assistência médica inicial.

Entre as causas de IAM (que, em geral, são do tipo STEMI), a mais comum é a isquemia, que é a redução abrupta ou interrupção do fluxo sanguíneo para um tecido devido a uma constrição ou obstrução arterial. Até o momento, existem dois principais tratamentos em Cardiologia para a retomada do fluxo sanguíneo em uma artéria previamente constrita ou obstruída: trombólise 
e angioplastia. A trombólise consiste na administração de fármacos capazes de dissolver a obstrução arterial de forma que o fluxo sanguíneo ao coração do paciente seja restabelecido efetivamente. A angioplastia, também chamada de intervenção coronariana percutanea primária (Primary Percutaneous Coronary Intervention - PPCI), é um procedimento altamente adotado, embora mais custoso, em casos de IAM isquêmico, que se baseia em uma intervenção cirúrgica para desbloqueio da artéria obstruída. Na literatura médica ${ }^{11}$ há um longo e recorrente debate a respeito de qual terapia - trombólise ou angioplastia - seria o mais adequado em casos de IAM. PPCI é a forma mais prevalente de tratamento de pacientes com IAM. Contudo, há em geral um consenso que: (i) reduzir o intervalo de tempo entre o primeiro contato com o sistema de atendimento e o início da terapia adotada (o chamado atraso do sistema) é essencial; (ii) a administração de trombólise pré-hospitalar é uma alternativa à angioplastia que pode produzir resultados semelhantes a um custo reduzido; e (iii) se o paciente é elegível ao tratamento com trombólise, então a combinação da administração da trombólise ainda em ambiente pré-hospitalar com a angioplastia como uma intervenção secundária após sua entrada no hospital alcança resultados proeminentes.

Essencialmente, a trombólise oferece o benefício de menor custo e de ser viável sua aplicação por emergencistas no cenário de primeiro atendimento da vítima de IAM. Entretanto a decisão de aplicação de trombólise requer uma avaliação criteriosa acerca da elegibilidade da vítima de IAM à trombólise. Tal avaliação tipicamente somente pode ser realizada por um especialista em cardiologia. Essa decisão especializada baseia-se na análise de um ECG além de informações sobre o histórico médico recente do paciente. Este é o ponto crucial onde surge o problema ao qual o sistema de SIS desenvolvido se posiciona. É pouco usual que o especialista esteja disponível no local do primeiro atendimento à vítima de
IAM, logo isso faz com que as taxas de administração de trombólise em ambiente pré-hospitalar sejam abaixo das recomendadas. Estudos recentes ${ }^{13}$ têm demonstrado a eficácia da adoção de soluções de telemedicina para casos de IAM neste contexto.

Dada esta motivação, nossa primeira experiência em SIS foi o desenvolvimento ad hoc de um sistema de teleconsulta para o suporte remoto em casos de IAM. Esse sistema permite a interação remota entre emergencistas, que fornecem o primeiro atendimento médico emergencial em ambiente pré-hospitalar a vítimas de IAM, e um cardiologista disponível em uma unidade coronariana. O sistema desenvolvido é chamado de AToMS $^{7}$, um acrônimo para AMI Teleconsultation \& Monitoring System. O AToMS utiliza-se de tecnologias ubíquas (sem fio e móveis) para possibilitar um serviço de telemedicina no local do primeiro atendimento à vítima de IAM, permitindo a emergencistas e cardiologistas: (i) trocar informações sobre um paciente vítima de IAM e decidir a respeito da elegibilidade do mesmo ao tratamento por trombólise dentro de uma janela adequada de tempo; e (ii) trocar informações complementares sobre a condição do paciente (incluindo ECGs complementares) enquanto este é deslocado a uma unidade coronariana.

O sistema AToMS visa a cobertura não somente em unidades convencionais de emergência, mas também virtualmente em qualquer lugar com cobertura de comunicação sem fio onde um paciente com IAM possa ser abordado por uma equipe móvel de emergência ou serviço de ambulância. Para alcançar esse objetivo, o sistema faz uso de diferentes tecnologias de interconexão para aumentar sua ubiquidade. A intervenção humana no AToMS é tipicamente restrita às solicitações de teleconsulta por parte dos emergencistas e as respostas a tais teleconsultas por parte dos cardiologistas. Uma solicitação de teleconsulta é realizada enviando uma solicitação de análise acerca de um paciente com IAM que 
inclui um ou mais registros de ECG digitalizados e informações clínicas recém-coletadas pelos emergencistas sobre o paciente. O sistema então envia a solicitação de análise do paciente para um cardiologista, que pode então decidir adequadamente sobre a elegibilidade ou não do paciente para tratamento antecipado em ambiente pré-hospitalar através de trombólise.

A Figura 1 fornece uma visão geral do fluxo de comunicação do AToMS, estruturado de acordo com um estilo arquitetural cliente-servidor.

O Servidor de Coordenação (Coordination Server CS) se ocupa da troca de informações entre emergencistas e cardiologistas. O CS recebe solicitações de análise enviadas pelos emergencistas usando uma interface cliente e as encaminha para o cardiologista conectado no sistema na outra interface cliente. Ao fazê-lo, o CS armazena toda a troca de informações em uma base de dados, permitindo auditoria posterior.

O lado cliente do sistema é composto de duas aplicações diferentes, que configuram os dois extremos do sistema. Uma aplicação é usada pelos emergencistas de um lado e outra aplicação é usada por cardiologistas do outro lado. Os emergencistas usam uma aplicação que executa sobre um dispositivo móvel capaz de comunicação sem fio (p.ex. $3 \mathrm{G}$ ou Wi-Fi) conectado através de uma interface USB com um dispositivo de aquisição de ECG proprietário. Essa aplicação permite aos emergencistas:
- Preencher um registro eletrônico do paciente (REP)[1] com (i) a informação clínica do paciente (p.ex. ritmo cardíaco, informação sobre critérios de exclusão à trombólise, etc); e (ii) um breve histórico médico do paciente recém-coletado por anamnese (p.ex. histórico de angioplastia, hipertensão, diabetes, etc);

- Obter um registro ECG do paciente (com 12 derivações ou menos) codificado como um arquivo pelo dispositivo ECG a ser anexado ao REP;

- Transmitir todos esses dados através de uma conexão sem fio (p.ex. 3G), logo definindo o lançamento de uma solicitação de análise no sistema, possivelmente seguido por mensagens de chat a um cardiologista disponível.

O CS então encaminha a solicitação de análise e as mensagens de chat ao cardiologista disponível no sistema presente na unidade coronariana mais próxima (o sistema permite o envolvimento de mais de uma unidade coronariana). Note que ao menos um cardiologista precisa estar conectado ao AToMS por unidade coronariana, do contrário a teleconsulta não faz sentido. A interface do cardiologista é acessada através de uma aplicação web que executa sobre um navegador web padrão. A aplicação recebe da CS uma lista de solicitações de análise pendentes, o que permite ao cardiologista analisar e responder a múltiplas solici-
Figura 1:

Visão geral do sistema AToMS.

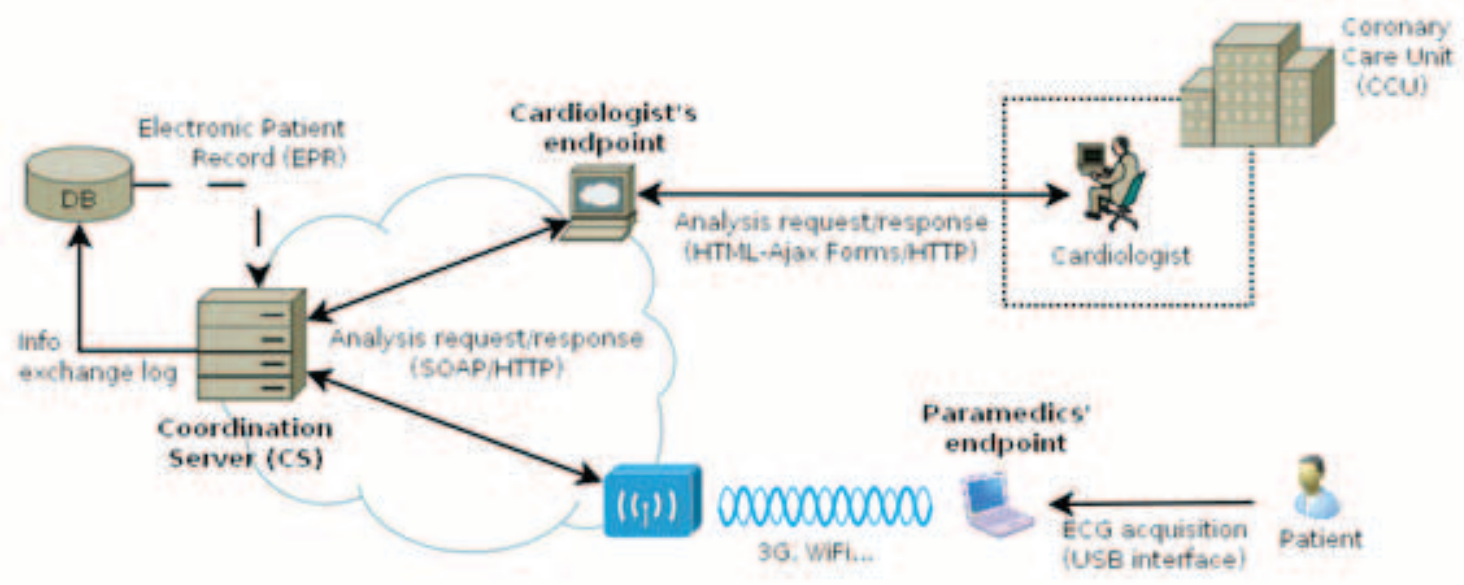


tações simultaneamente. Cada solicitação de análise é transmitida pelo CS ao cardiologista por HTTP assim que este se conecta ao sistema, e então é exibida como uma página HTML. Essa página contém:

- O REP e os link(s) para download dos registros ECG coletados pelos emergencistas para que o cardiologista analise;

- Um formulário web que molda o relatório médico que o cardiologista deve atribuir à solicitação. Isso inclui a indicação se a trombólise deve ou não ser ministrada ao paciente assim como a gravidade da condição do paciente de acordo com a avaliação;

- Um canal de chat para permitir comunicação em tempo-real entre os emergencistas e o cardiologista.

Todo relatório médico (isto é, o formulário web preenchido) atribuído pelo cardiologista é enviado ao CS, o qual por sua vez o repassa ao emergencista. Registros de ECG adicionais juntamente com o recurso do canal de chat permitem ao cardiologista obter do emergencista a condição corrente do paciente enquanto este está sendo deslocado à unidade coronariana. Isso fornece à equipe da unidade coronariana informações da situação do paciente ainda no cenário pré-hospitalar de forma que a equipe possa se preparar para o tratamento mais adequado subsequente para este paciente na unidade coronariana e aplicá-lo tão logo o paciente chegue.

Uma descrição detalhada do sistema AToMS pode ser encontrada em Correa et $\mathbf{a l}^{7}$. A provisão de qualidade de serviço (QoS) para um sistema de informação em saúde é fundamental para o funcionamento do sistema, quando há aplicações concorrentes na rede. Uma análise do uso de redes mesh com QoS como infraestrutura de comunicação para o sistema AToMS foi realizada e relatada em Silva et $\mathrm{al}^{14}$. Nesse experimento, foram realizados diversos testes práticos do sistema AToMS usando a rede mesh da Universidade
Federal Fluminense (UFF) em Niterói-RJ, com e sem tráfego concorrente, para medir a vazão, a perda e o atraso de pacotes. Foram considerados dois conjuntos de cenários de uso do sistema AToMS: sem a configuração de parâmetros de QoS, e com parâmetros de QoS, usando a ferramenta TC MESH (Traffic Control For Mesh Networks). Os testes foram realizadas com o auxílio das ferramentas Wireshark e IPERF.

\section{Experiência em Desenvolvimento Dirigido por Modelos de SIS}

Recentemente, tem havido considerável atenção sobre o potencial de Desenvolvimento Dirigido por Modelos $(D D M)^{15}$ no suporte ao desenvolvimento de SIS. De forma geral, as iniciativas existentes advogam o uso do padrão MDA (Model-Driven Architecture)' . Conforme descrito por Ragupathi e Umar ${ }^{5}$, a abordagem MDA viabiliza o desenvolvimento de SIS: (i) com baixo custo e time-to-market; (ii) interoperáveis; (iii) independentes de plataforma; e (iv) melhor manuteníveis. Contudo, em nossa visão, há dois desafios principais a serem ainda tratados para que DDM alcance um nível maior de interoperabilidade, manutenibilidade e reúso no desenvolvimento de SIS:

\section{- Complexidade na modelagem dos dados}

clínicos. Nossa experiência prévia no desenvolvimento de SIS $^{7}$ mostra que mudanças na modelagem de dados clínicos são constantes devido à complexidade de transcrever o conhecimento do profissional médico para dentro do sistema. Essa complexidade pode se traduzir em problemas de manutenibilidade e interoperabilidade a médio e longo prazo. Como consequência, a modelagem dos dados clínicos acaba exigindo atenção demasiada da equipe de desenvolvimento, sendo que constitui apenas uma parcela do esforço de desenvolvimento total de um SIS. 
- Modelagem arquitetural ad-hoc. Técnicas de projeto arquitetural são especialmente úteis no domínio de SIS. Nesse sentido, a abordagem MDA é capaz de facilitar o reúso de uma boa arquitetura, mas não auxilia o projeto de novas boas arquiteturas. Tipicamente, sistemas dentro de uma mesma família de SIS (p.ex., emergência pré-hospitalar, vigilância epidemiológica, monitoramento intra-hospitalar de pacientes) empregam estilos arquiteturais semelhantes (p.ex., cliente-servidor, publisher-subscriber, peer-to-peer). Defendemos que esses estilos devem poder ser melhor explorados pelas equipes de desenvolvimento visando projetos arquiteturais que propiciem manutenibilidade e reúso de longo prazo.

Esses dois desafios têm nos motivado a desenvolver um método de DDM para SIS mais abrangente que os baseados somente na abordagem MDA. Nosso método tem como objetivos:

- Transversalizar modelos arquiteturais e de dados clínicos, de modo que especialistas em saúde não se importem em como os dados são processados, armazenados e transportados, e que arquitetos de software não se importem em como os dados são representados em SIS;

\section{- Dualizar modelos de dados}

clínicos, para separar a modelagem computacional da modelagem médica, o que permite reduzir o esforço da equipe de desenvolvimento na manutenção de SIS;
- Estilizar modelos arquiteturais, para capturar características comuns dentro de famílias de SIS como artefatos reusáveis de projeto.

Para dar suporte ao método proposto, temos desenvolvido recentemente um ferramental de software inovador, que é apresentado na Seção de Resultados. O estado atual de desenvolvimento do ferramental e as perspectivas futuras de aplicação desse ferramental são apresentados na Seção de Conclusão.

\section{Resultados}

O ferramental proposto que encontra-se em desenvolvimento é apresentado na Figura 2. Todas as ferramentas ilustradas estão sendo prototipadas como plugins para o ambiente Eclipse e são detalhadas adiante.

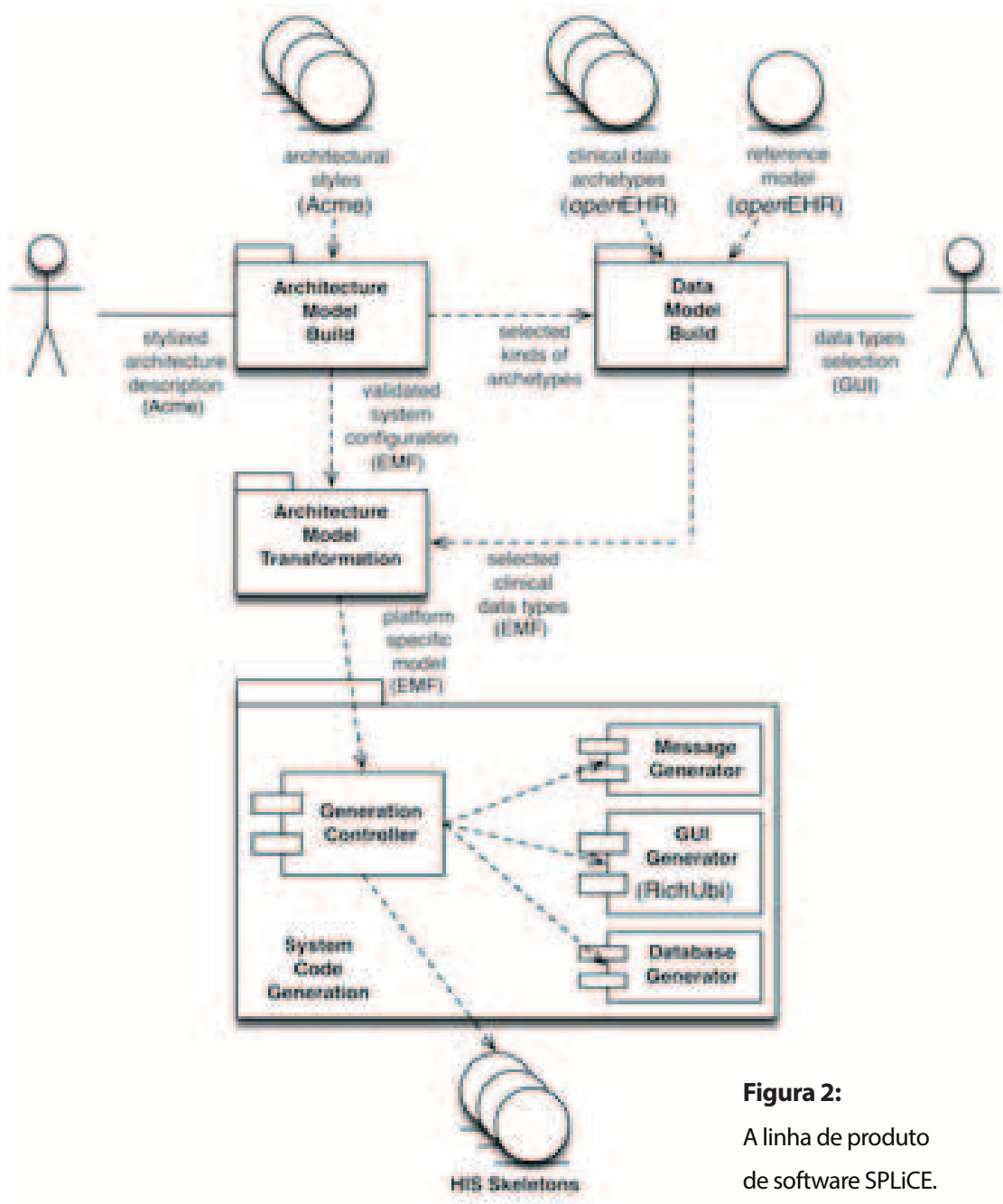




\section{Transversalização de Modelos}

Para transversalizar modelos arquiteturais e de dados clínicos, consideramos dois atores diferentes no método proposto: o especialista de domínio e o arquiteto de software. O primeiro especifica modelos de dados clínicos de SIS (vide Subseção "Modelagem de Dados Clínicos") e o segundo modelos arquiteturais de SIS (vide Subseção "Modelagem Arquitetural"). Esses dois modelos são transformados, conforme descrito nas subseções seguintes, em formatos compatíveis com o metametamodelo ECore do EMF (Eclipse Modeling Framework) e passados à ferramenta Architecture Model Transformation. Essa ferramenta implementa um motor de composição e transformação de modelos baseado no EMF que gera um modelo de plataforma específica (Platform Specific Model - PSM) também baseado no EMF, a partir do qual esqueletos de SIS são gerados (vide Subseção "Geração de Código").

\section{Modelagem de Dados Clínicos}

Para dualizar modelos de dados clínicos, adotamos no método proposto o conjunto de especificações openEHR ${ }^{16}$. No openEHR, a tarefa da modelagem da informação é mantida separada daquela da modelagem de dados clínicos. Modeladores de informação definem um modelo de referência, sobre o qual comitês multidisciplinares de analistas de sistemas e médicos trabalham na modelagem dos dados clínicos e produzem os chamados arquétipos de dados clínicos, que são basicamente conjuntos de restrições que restringem os tipos de dados definidos no modelo de referência. Por exemplo, no openEHR "Pressão sanguínea” é um arquétipo com tipos de dados como "Sistólica" e "Diastólica", que definem restrições sobre os valores aceitos pelo tipo de dados mais geral "Quantidade" no modelo de referência.
No ferramental de software proposto, ambos o modelo de referência e os arquétipos de dados clínicos das especificações openEHR servem como entrada para a ferramenta Data Model Build. Essa ferramenta também recebe da ferramenta Architecture Model Build (vide Subseção "Modelagem Arquitetural") uma indicação sobre quais tipos de arquétipos de dados clínicos são de interesse - observação, instrução, avaliação ou ação - para a família específica de SIS da qual o sistema a ser gerado faz parte. A partir dessas entradas, a ferramenta Data Model Build gera um modelo completo de dados clínicos. Esse modelo é repassado para a ferramenta Data Model Transformation, que: (i) permite que o especialista selecione os tipos de dados no modelo de dados clínicos completo que são de interesse para o SIS específico a ser gerado - por exemplo, o tipo de dado "Muscle Tone" é crucial para obstetras (devido ao método Apgar), mas não faz sentido para serviços de emergência para pacientes de infarto do miocárdio; e (iii) traduz os tipos de dados selecionados em um formato compatível com o EMF.

\section{Modelagem Arquitetural}

Para estilizar modelos arquiteturais, adotamos no método proposto a linguagem de descrição arquitetural Acme. Acme é uma notação construída para o propósito específico do intercâmbio de modelagem arquitetural. Acme oferece construções de alto nível como componentes, conectores e configurações - que incorporam informações sobre a estrutura de um sistema ao mesmo tempo que permitem abstrair detalhes da implementação do mesmo. Acme também provê suporte para estilos arquiteturais ${ }^{17}$, os quais prescrevem tipos de construções e restrições na composição dos mesmos. Por exemplo, um estilo "Pipeline" pode incluir um tipo de componente "Filter" e um tipo de conector 
"Pipe" além de restrições que impedem composições circulares de tais construções em uma configuração de sistema. Uma configuração seguindo um estilo específico em Acme pode assim ser vista como uma instância de tal estilo.

No ferramental de software proposto, estilos arquiteturais descritos em Acme servem como entrada para a ferramenta Architecture Model Build. Tais estilos definem regras para a configuração dos sistemas pertencentes a diferentes famílias de SIS - até o momento temos nos dedicado à definição de estilos Acme para sistemas de emergência pré-hospitalar e vigilância sindrômica. A ferramenta Architecture Model Build: (i) valida uma configuração de sistema especificada por um arquiteto de software de acordo com as regras de estilo da família SIS pretendida; (ii) indica à ferramenta Data Model Build os tipos de arquétipos de dados clínicos de interesse para essa família - por exemplo, sistemas de emergência manipulam em sua maioria dados de observação e instrução, enquanto que sistemas de vigilância estão normalmente interessados em dados de observação e avaliação; e (iii) traduz a configuração validada de sistema em um formato compatível com o EMF.

\section{Geração de Código}

A ferramenta System Code Generation implementa um motor de transformação baseado no EMF que gera esqueletos de código para SIS a partir de um PSM exportado pela ferramenta Architecture Model Transformation. A ferramenta System Code Generation é composta por quatro componentes. O componente Generation Controller analisa o PSM e invoca um dos três componentes geradores específicos dependendo da parte do PSM que está sendo analisada: (i) o componente Message Generator para a geração de entidades de protocolo e formatos de mensagens (p.ex. definições WSDL, stanzas
XMPP); (ii) o componente GUI Generator, que é baseado no RichUbi ${ }^{18}$, uma ferramenta também baseada no EMF para geração de código para interfaces gráficas de usuário; e (iii) o componente Database Generator para a geração de código específico de banco de dados (p.ex., esquemas, mapeamentos objeto/relacional).

\section{Discussão}

Recentemente, considerável atenção tem sido voltada para o potencial de DDM em geral, e o padrão MDA em particular, para a superação dos fatores inibidores ao desenvolvimento de sistemas de informação em saúde (SISs) manuteníveis.

Lopez e Blobel ${ }^{19}$ discutem diversas metodologias de engenharia de software (p.ex., RUP), linguagens de modelagem (p.ex., UML), arcabouços arquiteturais (p.ex. MDA, RM-ODP) e padrões de dados em saúde (p.ex., HL7, openEHR) no intuito de alcançar uma configuração viável para permitir uma interoperabilidade semântica na área de saúde. Nesse sentido, vale ressaltar a dificuldade existente em integrar e evoluir artefatos proprietários, tais como RUP e HL7. Em um caminho distinto, Janamanchi et al. ${ }^{20}$ discutem o potencial de disseminação de artefatos abertos, tais como o EMF e as especificações openEHR. Os autores concluem que existe uma comunidade de software livre ativa e florescente que está focada em informática em saúde.

Com base nessa breve revisão da literatura recente, nós enfatizamos que a aplicação de DDM na área de informática em saúde está em progresso, mas as iniciativas existentes estão em geral ainda restritas à abordagem MDA padrão.

A abordagem MDA depende do uso de regras de transformação (semi)automáticas para traduzir um modelo independente de plataforma (Platform Independent Model - PIM) em diferentes modelos de plataforma específica (Platform Specific Model - PSM) 
e então gerar código para o último. Isso permite que se gerencie mudanças funcionais diretamente no PIM para então gerar PSMs e código, ao invés de (na pior das hipóteses) lidar com mudanças como acertos no código. Isso significa uma melhor gerência do chamado roundtrip problem, ou seja, lidar com múltiplos artefatos que representam diferentes níveis de abstração no ciclo de vida de desenvolvimento de tal forma a evitar redundância e inconsistência. Essa característica constitui um importante passo para reduzir o alto custo de desenvolvimento de SIS.

A abordagem MDA por si só pode ser um recurso valioso para o desenvolvimento de SIS com a separação entre processos de saúde e tecnologia. Entretanto, isso deve ser incrementado a fim de se melhorar a reutilização em termos da modelagem independente de aplicação. No caso da área de saúde, particularmente, modelar dados clínicos bem como arquiteturas de software caso a caso, de forma ad-hoc, pode ser contraprodutivo.

Por um lado, nossa experiência com o desenvolvimento do sistema AToMS ${ }^{7}$ indica que a modelagem de dados clínicos requer atenção demasiada da equipe de desenvolvimento. Por outro lado, a modelagem arquitetural almejada de um SIS - por exemplo, para prover usuários com fácil interação com sistemas clínicos permanentemente disponíveis - está longe de ser uma tarefa trivial. Portanto, sejam dirigidos por modelos ou não, para esses sistemas serem "a prova de futuro", um projeto arquitetural cuidadoso é importante, se não indispensável. Nesse caso, MDA é capaz de facilitar o reúso de uma boa arquitetura, mas ainda não pode sistematicamente ajudar alguém a obter novas boas arquiteturas. Entretanto, pode-se vislumbrar no domínio de SISs padrões de comunicação e controle que sejam adequados a famílias específicas de sistemas. Por exem- plo, os requisitos de (quase) tempo-real em sistemas de vigilância sindrômica ou de atendimento médico emergencial não necessariamente importam em sistemas de vigilância epidemiológica. Nesse último, entretanto, a robustez e confiabilidade da informação disponível é de vital importância. Em consonância com essa linha de raciocínio, os princípios estabelecidos de arquitetura de software em geral, e de estilos arquiteturais ${ }^{17}$ em particular, produzem uma clara sinergia dentro da filosofia de DDM.

\section{Conclusão}

Neste documento, nós discorremos a respeito de um método que aplica DDM para entrelaçar modelos de dados clínicos e modelos arquiteturais de forma a gerar SIS manuteníveis. Nós também apresentamos nosso trabalho em andamento no desenvolvimento de uma linha de produto de software, chamada SPLiCE, que suporta tal método. De maneira geral, nós acreditamos que são promissoras as perspectivas para DDM representar um papel chave no futuro próximo da área de SIS.

Dentro do projeto atualmente em andamento, nós também temos elencado as similaridades e diferenças entre SIS de duas famílias principais: emergência préhospitalar e vigilância epidemiológica. Para sistemas de vigilância, por exemplo, um ponto comum consiste em que eles precisam permitir que alertas relacionados à saúde e as respostas correspondentes aconteçam de uma maneira altamente distribuída de forma a assegurar que tomadores de decisão cumpram adequadamente os programas e políticas do atendimento de saúde. Esse ponto comum leva à transmissão informal de idiomas arquiteturais entre os sistemas. No entanto, o desenvolvimento tecnológico fenomenal em computação 
móvel e redes na última década tornou-se um ponto essencial para a produção de uma nova família de SIS, a qual nós coletivamente chamamos de SIS ubíquos. Para sistemas de vigilância, em particular, tais tecnologias de comunicação sem fio são fundamentais para a provisão dos alertas e respostas no devido tempo. Essa escala temporal, entretanto, basicamente depende do cenário específico de aplicação das práticas de vigilância pretendidas. Cada cenário pode divergir em uma ou mais das seguintes características: (i) o método de detecção usado (ativo vs. passivo); (ii) a velocidade através da qual os dados precisam fluir através do sistema (imediato vs. rotina); (iii) a rapidez de resposta requerida (investigação imediata de casos ou agrupamentos de casos vs. análise de dados de maneira regular com subsequentes ajustes na política de controle). Isso leva a diferenças nos requisitos não-funcionais específicos que precisam ser tratados de acordo com cada SIS dessa família.

Como trabalho futuro, nós pretendemos validar a metodologia proposta e a linha de produto de software SPLiCE pela modelagem de SIS legados reais (p.ex. AToMS 7 ) e compará-los aos esqueletos de SIS gerados pela proposta.

\section{Agradecimentos}

Este trabalho foi parcialmente financiado com recursos do CNPq, FAPERJ e MCTI. Os autores agradecem a colaboração (i) dos cardiologistas Nelson A. de Souza e Silva (UFRJ) e Wilson B. Correa Filho (SAMU-RJ/UFRJ), e (ii) da epidemiologista Luciana T. Cavalini (UFF), respectivamente pelas informações acerca de atendimento emergencial de vítimas de infarto agudo do miocárdio e vigilância epidemiológica. Este artigo foi convidado para publicação no Jornal Brasileiro de Telessaúde após o capítulo de livro correspondente publicado no livro "GoldBook: Inovação Tecnológica em Educação e Saúde" ter sido selecionado entre os cinco melhores capítulos publicados.

\section{Referências}

1. Haux R. Medical informatics: past, present, future. Int J Med Inform. 2010;79(9):599-610. 2. Estrin D, Sim I. Open mHealth Architecture: An Engine for Health Care Innovation Science. 2010;330(5):759-60.

3. Cantrill SV. Computers in patient care: the promise and the challenge. Communications of the ACM. 2010;53(9):42-7.

4. Vest JR. More than just a question of technology: factors related to hospitals adoption and implementation of health information exchange. International journal of medical informatics. 2010 Dec;79(12):797-806.

5. Raghupathi W, Umar A. Exploring a model-driven architecture (MDA) approach to health care information systems development. Int J Med Inform. 2008 May;77(5):305-14.

6. Instituto Nacional de Ciência e Tecnologia Medicina Assistida por Computação Científica (INCT - MACC). Rio de Janeiro: 2009-2013 [acesso em 13 de abril de 2012]. Disponível em: http://macc.Incc.br.

7. Correa BSPM, Gonçalves BN, Teixeira IM, Gomes ATA, Ziviani A. AToMS: A Ubiquitous Teleconsultation System for Supporting AMI Patients with Pre-Hospital Thrombolysis. International Journal of Telemedicine and Applications; 2011:1-12. doi:10.1155/2011/560209

8. Job DH, Gomes ATA, Ziviani A. Health Systems for Syndromic and Epidemiological Surveillance. In: Telemedicine and E-Health Services, Policies and Applications: Advancements and Developments. IGI Global; 2012. p. 245-62.

9. Gomes ATA, Ziviani A, Correa BSPM, Teixeira IM, Moreira VM. SPLiCE: A Software Product Line for Healthcare. In: Proceedings of the 2nd ACM SIGHIT symposium on International health informatics - IHI'12. New York (NY) USA: ACM Press; 2012.

10. Canto JG. Use of Emergency Medical Services in Acute Myocardial Infarction and Subsequent Quality of Care: Observations From the National Registry of Myocardial Infarction. Circulation. 2002 Nov; 106(24):3018-23.

11. Danchin N, Durand E, Blanchard D. Pre-hospital thrombolysis in perspective. European heart journal. 2008 Dec;29(23):2835-42

12. McLean S, Wild S, Connor P, Flapan AD. Treating ST elevation myocardial infarction by primary percutaneous coronary intervention, in-hospital thrombolysis and prehospital thrombolysis. An observational study of timelines and outcomes in 625 patients. EmergMed J. 2011 Mar;28(3):230-6.

13. de Waure C, Cadeddu C, Gualano MR, Ricciardi W. Telemedicine for the Reduction of Myocardial Infarction Mortality: A Systematic Review and a Meta-Analysis of Published Studies. Telemed E Health. 2012 Apr;18(5):1-6.

14. Silva B, Gomes ATA, Ziviani A, Muchualuat-Saade DC. Provisão de QoS para um Sistema de Teleatendimento Médico Emergencial em Redes em Malha sem Fio. In: Anais do XXII Congresso Brasileiro de Engenharia Biomédica; 2010 Nov 21-25; Tiradentes, Minas Gerais.

15. Walderhaug S, Stav E, Mikalsen M. Experiences from model-driven development of homecare services: UML profiles and domain models. Models in Software Engineering. Berlin: Springer-Verlag; 2009. p.199-212.

16. Heard S, Beale T. OpenEHR Architecture Overview [on-line]. OpenEHR Foundation;2007 [acesso em 27/09/2012]. Disponível em: http://www.openehr.org/releases/1.0.1/ architecture/overview.pdf

17. Garlan D, Allen R, Ockerbloom J. Exploiting style in architectural design environments. ACM SIGSOFT Software Engineering Notes. 1994 Dec;19(5):175-88.

18. Cirilo CE, do Prado AF, de Souza WL, Zaina LAM. Model driven RichUbi. In: Proceedings of the 28th ACM International Conference on Design of Communication - SIGDOC'10. New York, New York, USA: ACM Press; 2010. p. 207.

19. Lopez DM, Blobel BGME. A development framework for semantically interoperable health information systems. Int J Med Inform. 2009 Feb;78(2):83-103.

20. Janamanchi B, Katsamakas E, RaghupathiW, GaoW. The state and profile of open source software projects in health and medical informaticsInt J Med Inform. 2009 Jul;78(7):457-72. 